\title{
Enaminones as building blocks in organic syntheses: on the reaction of 3-dimethylamino-2-propenones with malononitrile
}

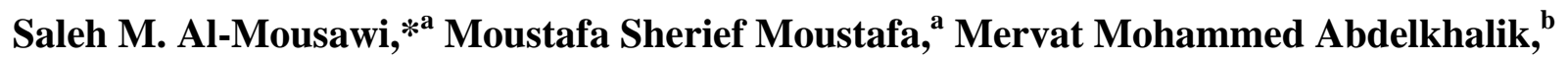 \\ and Mohamed Hilmy Elnagdi ${ }^{\text {a }}$ \\ ${ }^{a}$ Department of Chemistry, Faculty of Science; University of Kuwait: Safat; 13060: P.O. Box \\ 12613, Kuwait \\ ${ }^{b}$ Applied Science Department, College of Technological Studies, Public Authority for Applied \\ Education and Training, P.O. Box 5969 Safat, 13060, Kuwait \\ E-mail: saleh.almousawi@hotmail.com
}

\begin{abstract}
An X-ray crystal structure for the product of reacting enaminones and malononitrile in ethanol with piperidine conclusively established its structure as dienamides $\mathbf{4 a , b}$ that cyclize to 1,2dihydropyridine-3-carbonitriles 5 on treatment with acetic anhydride in the presence of hydrochloric acid. Reaction of $\mathbf{4 a}$ with a second mol equivalent of malononitrile afforded 2-[3(dimethylamino)-3-phenyl-2-propenylidene]malononitrile 7 that was also converted into 5, and to 5-phenyl-1H-pyrazole 9 via treatment with hydrazine hydrate. Reaction of enaminone 1a with ethyl cyanoacetate in the presence of piperidine afforded the pyranone derivative 12.
\end{abstract}

Keywords: Enaminones, malononitrile, dienonitrile, pyridones, dienodinitrile, and pyranone

\section{Introduction}

Enaminones are versatile building blocks in organic synthesis and their chemistry has received considerable interest. ${ }^{1}$ In the last decade we have reported efficient syntheses of polyfunctional molecules utilizing enaminones as starting materials. ${ }^{2-10}$ Our synthetic approaches have attracted attention. ${ }^{11-15}$ Sometime ago we reported that enaminones $\mathbf{1}$ react with malononitrile in refluxing ethanolic sodium ethoxide solution to yield alkoxypyridines $2 .{ }^{16}$ However, it was subsequently noted that in ethanolic piperidine solution compound 3 was produced. ${ }^{17}$ Recently Kappe et al. ${ }^{18}$ reported a 1,4-addition of malononitrile to enaminones using microwave heating. This led us to reconsider the structure assigned for 3 , as if it were correct it would be the only example of 1,2addition of an active methylene to an enaminone. Recently we ${ }^{19}$ concluded that $\mathbf{3}$ actually has structure 4 based on spectral data ( $c f$. Scheme 1). It seemed to us of value to further confirm this 
conclusion and to explore the chemistry of this newly formed diene. In addition, examining the behavior of other active methylenes towards $\mathbf{1 b}$ under similar conditions seemed of value.

\section{Results and Discussion}

Both 1a,b were reacted with malononitrile in ethanolic piperidine to yield products whose analytical and spectral data agree well with both the old structures 3a,b and revised ones 4a,b. Firstly we obtained an X-ray crystal structure for the product of reacting 1a with malononitrile in ethanolic piperidine. We assume that malononitrile initially undergoes 1,4 addition to the double bond in 1a,b yielding the adduct $\mathbf{A}$ that cyclizes into aminopyran $\mathbf{B}$ before undergoing a rearrangement to $\mathbf{4 a , b}$ via a 1,3-nitrogen shift. The X-ray data ${ }^{20}$ established the structure of 2cyano-5-(dimethylamino)-5-(furan-2-yl)-penta-2,4-dienamide $\mathbf{4 b}$, clearly confirming previous recent conclusion. ${ }^{19}$ It also shows that the molecule adapts the $(E, E)$ form indicated ( $c f$. Figure 1 and Table 1).

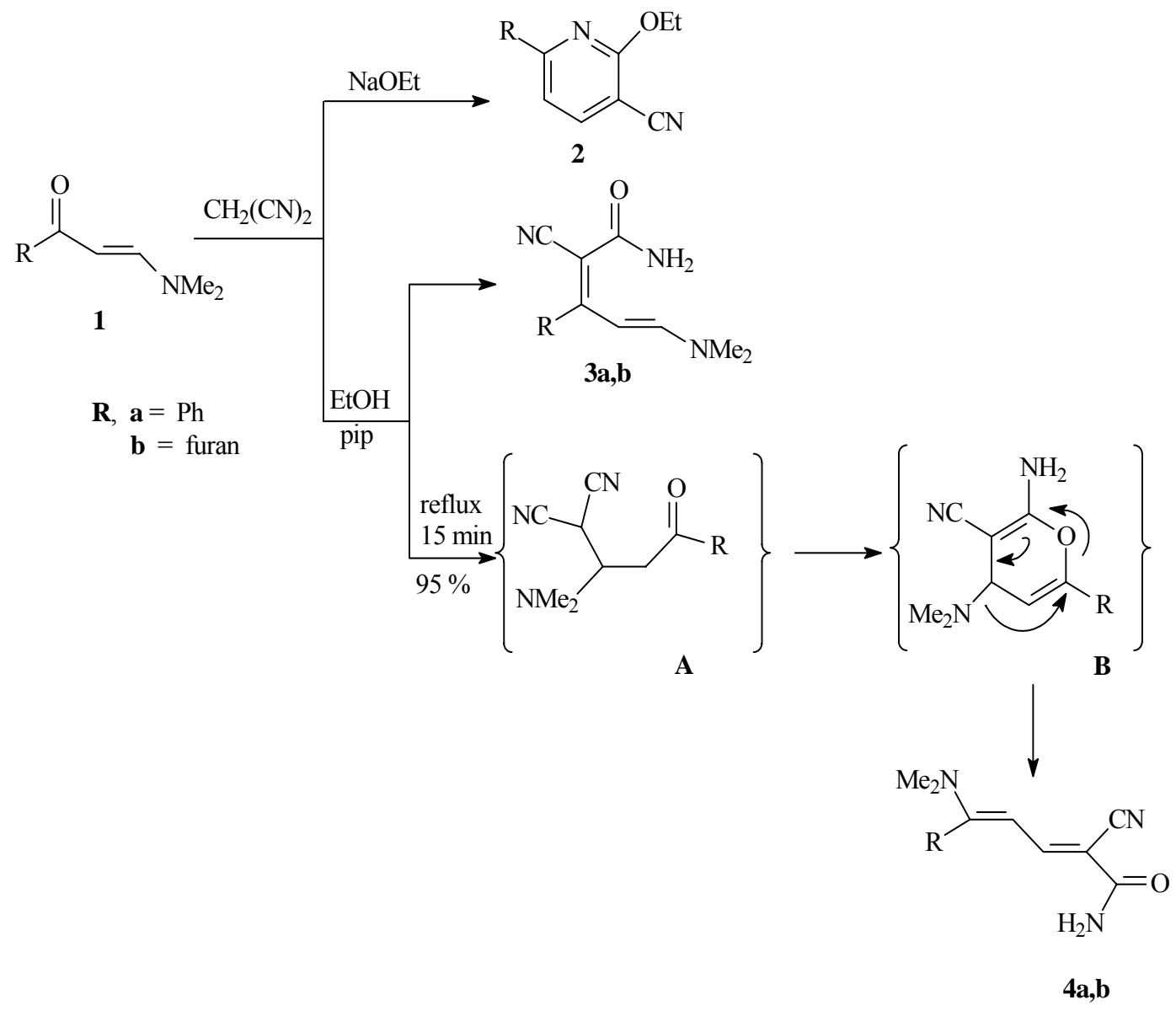

Scheme 1. Syntheses of dienonitriles $4 \mathbf{4 a}, \mathbf{b}$. 

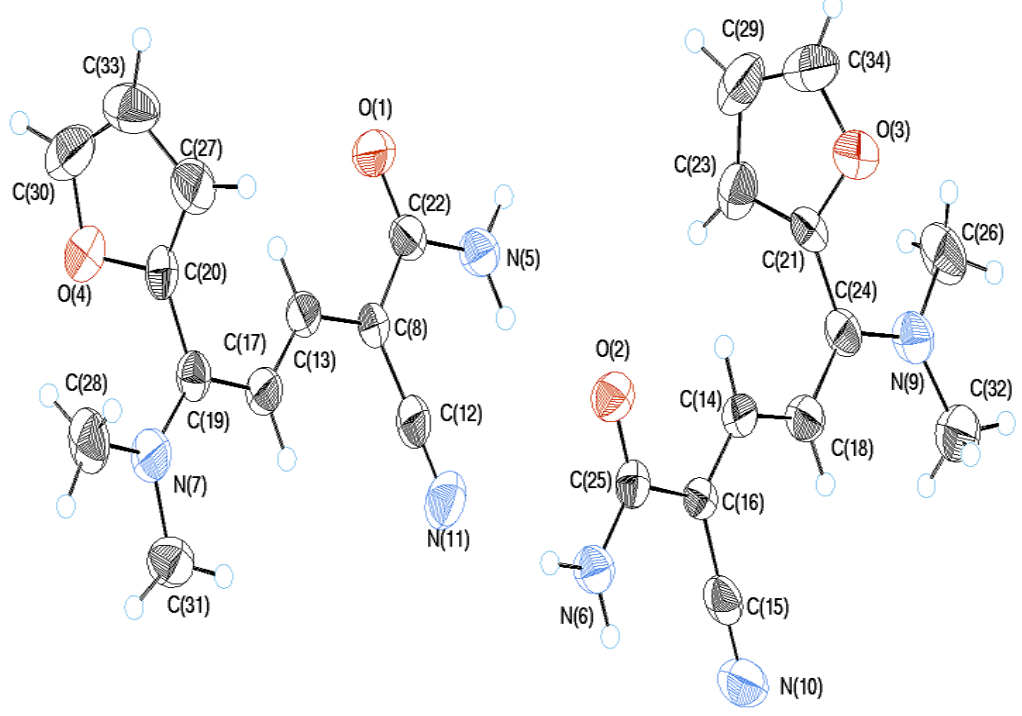

Figure 1. X-ray crystal structure of compound $4 \mathbf{b}$ 2-cyano-5-(dimethylamino)-5-(furan-2yl)penta-2,4-dienamide. Typical bond lengths and bond angles are reported in Table 1.

Table 1. Selected bond lengths and bond angles for compound $\mathbf{4 b}$

\begin{tabular}{llll}
\hline Bond lengths & \multicolumn{3}{c}{ Bond angles } \\
\hline Atoms numbers & $\begin{array}{l}\text { Geometric } \\
\text { parameters }\left(\AA,{ }^{\circ}\right)\end{array}$ & Atoms numbers & $\begin{array}{l}\text { Geometric } \\
\text { parameters }\left(\AA,{ }^{\circ}\right)\end{array}$ \\
\hline $\mathrm{N} 7-\mathrm{C} 19$ & $1.350(5)$ & $\mathrm{C} 19-\mathrm{N} 7-\mathrm{C} 28$ & $122.6(3)$ \\
$\mathrm{N} 7-\mathrm{C} 28$ & $1.469(5)$ & $\mathrm{C} 19-\mathrm{N} 7-\mathrm{C} 31$ & $121.4(3)$ \\
$\mathrm{N} 7-\mathrm{C} 31$ & $1.451(5)$ & $\mathrm{C} 28-\mathrm{N} 7-\mathrm{C} 31$ & $115.5(3)$ \\
$\mathrm{C} 8-\mathrm{C} 13$ & $1.372(5)$ & $\mathrm{C} 12-\mathrm{C} 8-\mathrm{C} 13$ & $119.8(3)$ \\
$\mathrm{C} 8-\mathrm{C} 22$ & $1.467(5)$ & $\mathrm{C} 12-\mathrm{C} 8-\mathrm{C} 22$ & $119.8(3)$ \\
$\mathrm{C} 13-\mathrm{C} 17$ & $1.406(5)$ & $\mathrm{C} 18-\mathrm{C} 8-\mathrm{C} 22$ & $120.3(3)$ \\
$\mathrm{C} 17-\mathrm{C} 19$ & $1.377(5)$ & $\mathrm{C} 8-\mathrm{C} 13-\mathrm{C} 17$ & $126.3(3)$ \\
& & $\mathrm{C} 13-\mathrm{C} 17-\mathrm{C} 19$ & $124.2(3)$ \\
& & $\mathrm{N} 7-\mathrm{C} 19-\mathrm{C} 17$ & $122.2(3)$ \\
& & $\mathrm{N} 7-\mathrm{C} 19-\mathrm{C} 20$ & $117.0(3)$ \\
& & $\mathrm{C} 17-\mathrm{C} 19-\mathrm{C} 20$ & $120.8(3)$ \\
\hline
\end{tabular}

Compound 4a could be converted into pyridone 5 upon treatment with acetic anhydride in the presence of hydrochloric acid whereas in $\mathrm{AcOH} / \mathrm{HCl}$ compound 6 was produced. It is logical to assume the hydrolysis of the initially formed $\mathbf{5}$ under this condition. Unexpectedly, reacting $\mathbf{4 a}$ with malononitrile in ethanolic piperidine resulted in the formation of the dienodinitrile 7 in an excellent yield $(95 \%)$. We assume that malononitrile initially added to $\mathbf{4 a}$ forming the Michael adduct 8 that then looses cyanoacetamide to yield 2-(3-(dimethylamino)-3- 
phenylallylidene)malononitrile 7 . Structure 7 was confirmed by an X-ray crystal structure ${ }^{16}$ determination ( $c f$. Figure 2).

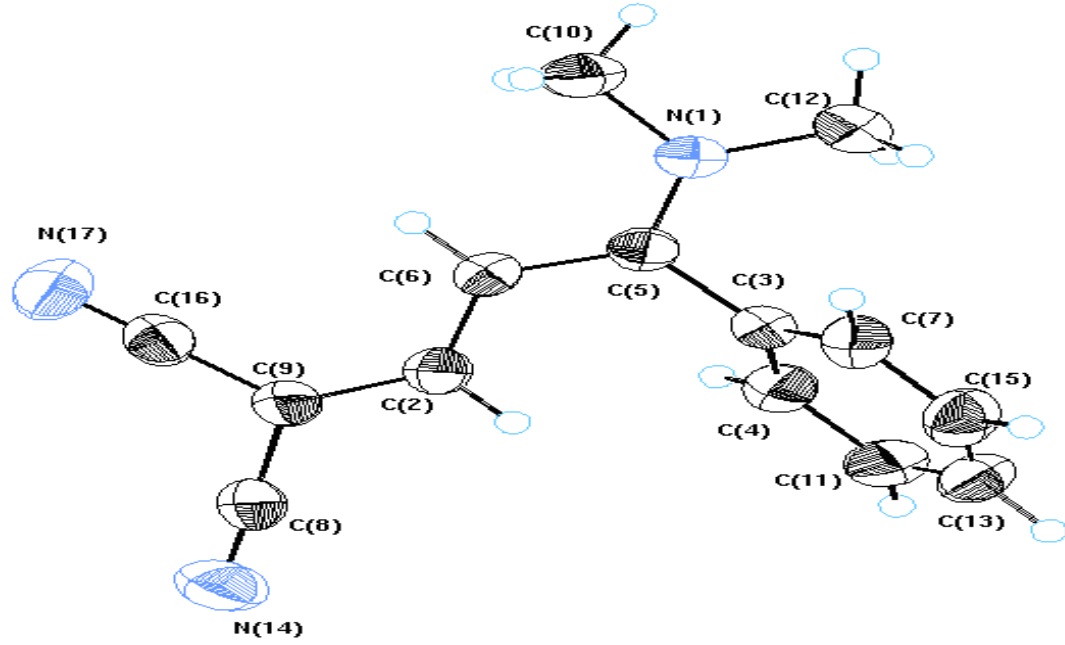

Figure 2. X-ray crystal structure of 2-(3-(dimethylamino)-3-phenylallylidene)-malononitrile 7.

The data clearly indicate that, the molecule adapts an E configuration. In Table 2 selected bond lengths and bond angles are reported.

Table 2. Selected bond lengths and bond angles for compound 7

\begin{tabular}{llll}
\hline Bond lengths & & Bond angles & \\
\hline $\begin{array}{l}\text { Atoms } \\
\text { numbers }\end{array}$ & $\begin{array}{l}\text { Geometric } \\
\text { parameters } \\
\left(\AA,^{\circ}\right)\end{array}$ & Atoms numbers & $\begin{array}{l}\text { Geometric } \\
\text { parameters }\left(\AA,{ }^{\circ}\right)\end{array}$ \\
& & & \\
\hline $\mathrm{N} 1-\mathrm{C} 5$ & $1.336(2)$ & $\mathrm{C} 5-\mathrm{N} 1-\mathrm{C} 10$ & $121.6(2)$ \\
$\mathrm{N} 1-\mathrm{C} 10$ & $1.466(2)$ & $\mathrm{C} 5-\mathrm{N} 1-\mathrm{C} 12$ & $123.35(15)$ \\
$\mathrm{N} 1-\mathrm{C} 12$ & $1.467(2)$ & $\mathrm{C} 10-\mathrm{N} 1-\mathrm{C} 12$ & $114.89(14)$ \\
$\mathrm{C} 2-\mathrm{C} 6$ & $1.399(2)$ & $\mathrm{C} 6-\mathrm{C} 2-\mathrm{C} 9$ & $126.7(2)$ \\
$\mathrm{C} 2-\mathrm{C} 9$ & $1.379(2)$ & $\mathrm{N} 1-\mathrm{C} 5-\mathrm{C} 3$ & $117.7(2)$ \\
$\mathrm{C} 3-\mathrm{C} 5$ & $1.494(2)$ & $\mathrm{N} 1-\mathrm{C} 5-\mathrm{C} 6$ & $122.4(2)$ \\
$\mathrm{C} 5-\mathrm{C} 6$ & $1.391(2)$ & $\mathrm{C} 3-\mathrm{C} 5-\mathrm{C} 6$ & $119.91(15)$ \\
& & $\mathrm{C} 2-\mathrm{C} 6-\mathrm{C} 5$ & $122.3(2)$ \\
& & $\mathrm{C} 2-\mathrm{C} 9-\mathrm{C} 8$ & $121.1(2)$ \\
& & $\mathrm{C} 2-\mathrm{C} 9-\mathrm{C} 16$ & $123.0(2)$ \\
& & $\mathrm{C} 8-\mathrm{C} 9-\mathrm{C} 16$ & $115.8(2)$ \\
\hline
\end{tabular}


Compound 7 was readily converted into 5 on refluxing with $\mathrm{Ac}_{2} \mathrm{O} / \mathrm{HCl}$. Reacting 7 with hydrazine hydrate afforded 5-phenylpyrazole $9^{16}$ (cf. Scheme 2).<smiles>CN(C)C1=CC(C(C#N)C#N)C(C#N)CCCCC1</smiles>

8<smiles>CN(C)/C(=C\C=C(C#N)C#N)c1ccccc1</smiles>

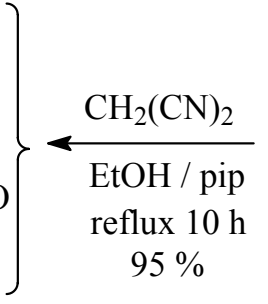

\section{$4 a$}

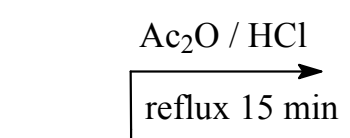

$85 \%$

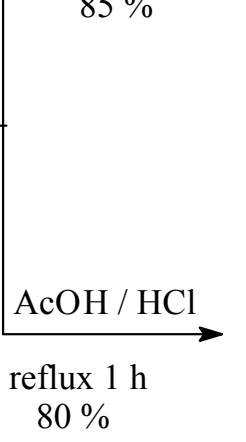

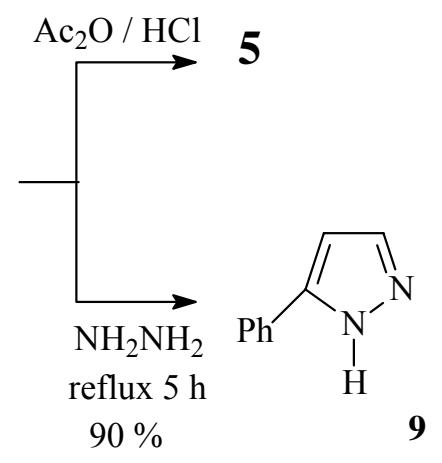

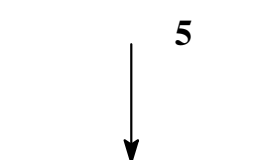<smiles>NC(=O)c1ccc(-c2ccccc2)[nH]c1=O</smiles>

Scheme 2. Syntheses of pyridone 5 and dienodinitrile 7.

In the light of the established behavior of $\mathbf{1}$ toward malononitrile we inspected its behavior toward other active methylene reagents. Reaction of $1 \mathbf{a}$ with cyanoacetic acid in $\mathrm{Ac}_{2} \mathrm{O}$ afforded the pyranone 10 while reaction with ethyl cyanoacetate in ethanolic piperidine gave a product that could be assigned structure $\mathbf{1 1}$ or its isomer 12, based on the analytical and the spectral data. ${ }^{1} \mathrm{H}$ NMR data indicated the presence of two doublet protons at $\delta=5.81$ and $\delta=7.71 \mathrm{ppm}$ with $J$ $=12 \mathrm{~Hz}$, typical for adjacent cis protons. Thus structure $\mathbf{1 2}$ is preferred over 11. It is assumed that the initially formed Michael adduct 13 cyclized to 14 and that the latter then undergoes a 1,2-dimethylamino group shift accompanying the elimination of the cyano group to yield the final isolable 12. The latter was readily converted into compound $\mathbf{9}$ when reacted with hydrazine hydrate. Coupling 3-(dimethylamino)-6-phenyl-2H-pyran-2-one 12 with benzenediazonium chloride afforded the known phenylhydrazone 17. It is believed that initially $\mathbf{1 5}$ is formed then undergoes ring opening to $\mathbf{1 6}$ and then is hydrolyzed under coupling reaction conditions to yield phenylhydrazone 17 ( $c f$. Scheme 3). At the moment we are looking into the behavior of $\mathbf{1 b}$ with a variety of other enaminones. 

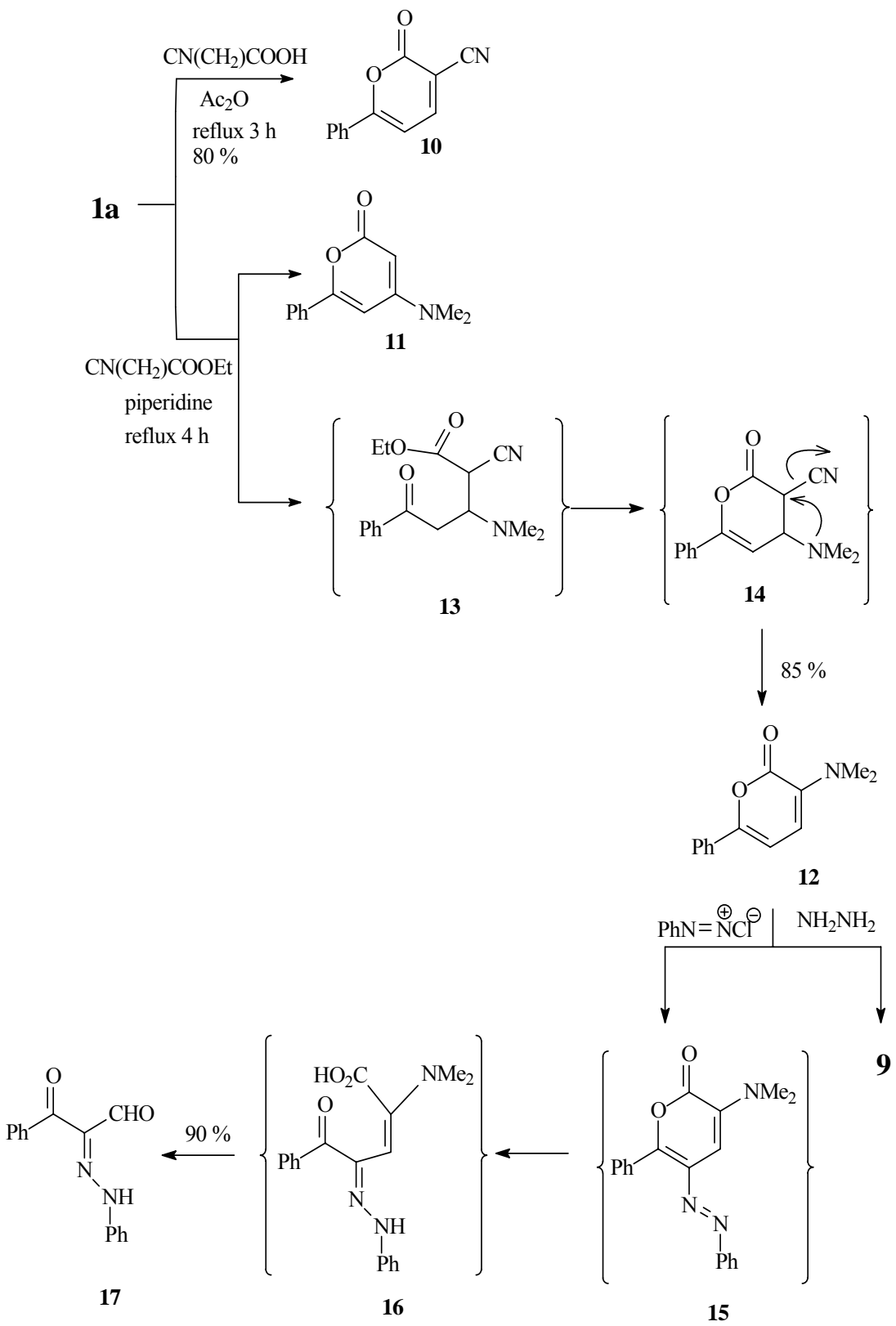

Scheme 3. Syntheses of pyranone 10 and phenylhydrazone 17.

\section{Conclusions}

We could thus revise, with certainity, the reported structures for products of reacting enaminones 1a with malononitrile and proved that the product is the diene 2-cyano-5-(dimethylamino)-5phenylpenta-2,4-dienamide 4a. 


\section{Experimental Section}

General Procedures. All melting points are uncorrected and were determined on a Sanyo (Gallaenkamp) instrument. Infrared spectra were recorded in $\mathrm{KBr}$ on a Perkin-Elmer 2000 FTIR system. ${ }^{1} \mathrm{H}-\mathrm{NMR}$ and ${ }^{13} \mathrm{C}-\mathrm{NMR}$ spectra were determined on a Bruker DPX spectrometer operating at $400 \mathrm{MHz}$ for ${ }^{1} \mathrm{H}-\mathrm{NMR}$ and $100 \mathrm{MHz}$ for ${ }^{13} \mathrm{C}-\mathrm{NMR}$ using in $\mathrm{CDCl}_{3}$ or DMSO-d6 as solvents and TMS as internal standard; chemical shifts are reported in $\delta(\mathrm{ppm})$. Mass spectra were measured on VG Autospec Q MS 30 and MS 9 (AEI) spectrometers, with EI 70 EV. Elemental analyses were measured by means of LEOCHNS-932 Elemental Analyzer. General purpose silica gel on polyester $20 \times 20 \mathrm{~cm}$ TLC plates with UV indicator were used in TLC experiments.

Synthesis of 2-cyano-5-(dimethylamino)-5-phenylpenta-2,4-dienamide (4a). A mixture of 1a $(1.75 \mathrm{~g}, 0.01 \mathrm{~mol})$ and malononitrile $(0.66 \mathrm{~g}, 0.01 \mathrm{~mol})$ in ethanol $(15 \mathrm{~mL})$ as a solvent was treated with piperidine (5 drops). The reaction mixture was refluxed for $15 \mathrm{~min}$, and then was poured into ice-water. The solid, so formed, was collected by filtration and recrystallized from $N, N$-dimethylformamide (DMF) to give yellow crystals; yield $95 \%$; mp $259-60{ }^{\circ} \mathrm{C}$. Anal. Calcd for $\mathrm{C}_{14} \mathrm{H}_{15} \mathrm{~N}_{3} \mathrm{O}$ (241.29): C, 69.69; H, 6.27; N, 17.41\% Found: C, 69.78; H, 5.93; N, 17.48\% IR $(\mathrm{KBr}): v_{\max }=333,3293\left(\mathrm{NH}_{2}\right), 2193(\mathrm{CN}), 1647(\mathrm{CO}) ;{ }^{1} \mathrm{H}$ NMR $\left(400 \mathrm{MHz}, \mathrm{DMSO}-d_{6}\right): \delta$, ppm $=2.50\left(\mathrm{~s}, 3 \mathrm{H}, \mathrm{CH}_{3}\right), 2.78\left(\mathrm{~s}, 3 \mathrm{H}, \mathrm{CH}_{3}\right), 5.63(\mathrm{~d}, J=12 \mathrm{~Hz}, 1 \mathrm{H}, \mathrm{CH}), 6.82\left(\mathrm{~s}, 2 \mathrm{H}, \mathrm{NH}_{2}, \mathrm{D}_{2} \mathrm{O}\right.$ exchangeable), $7.11(\mathrm{~d}, J=12 \mathrm{~Hz}, 1 \mathrm{H}, \mathrm{CH}), 7.25-7.55(\mathrm{~m}, 5 \mathrm{H}, \mathrm{Ar}-\mathrm{H}) ;{ }^{13} \mathrm{C}$ NMR $(100 \mathrm{MHz}$, DMSO- $\left.d_{6}\right): \delta, \mathrm{ppm}=165.55,164.71,153.38,133.92,129.65,128.83(2 \mathrm{C}), 128.78(2 \mathrm{C}), 119.01$, 99.03, 87.53, 66.38, 42.01. MS: m/z (\%) $241\left(\mathrm{M}^{+}, 100\right), 223$ (15), 197 (75), 182 (15), 158 (40), 127 (15), 118 (35), 91 (10), 77 (20).

Synthesis of 2-oxo-6-phenyl-1,2-dihydropyridine-3-carbonitrile (5). A mixture of compound 4a $(2.41 \mathrm{~g}, 0.01 \mathrm{~mol})$ and acetic anhydride $\left(\mathrm{Ac}_{2} \mathrm{O}\right)(15 \mathrm{~mL})$ as a solvent was treated with hydrochloric acid $(5 \mathrm{~mL})$, then the mixture was refluxed for $15 \mathrm{~min}$. The reaction mixture was cooled and poured into ice-water. The solid, so formed, was collected by filtration and recrystallized from ethanol to give faint yellow product; yield $85 \%$; mp $295-97^{\circ} \mathrm{C}$. Anal. Calcd for $\mathrm{C}_{12} \mathrm{H}_{8} \mathrm{~N}_{2} \mathrm{O}$ (196.21): C, 73.46; H, 4.11; N, 14.28\% Found: C, 73.09; H, 3.97; N, 14.25\% IR $(\mathrm{KBr}): v_{\max }=3151(\mathrm{NH}), 2222(\mathrm{CN}), 1648(\mathrm{CO}) ;{ }^{1} \mathrm{H}$ NMR $\left(400 \mathrm{MHz}, \mathrm{DMSO}-d_{6}\right): \delta, \mathrm{ppm}=$ 6.77 (d, $J=8 \mathrm{~Hz}, 1 \mathrm{H}, \mathrm{CH}), 7.53-7.82(\mathrm{~m}, 5 \mathrm{H}, \mathrm{Ar}-\mathrm{H}), 8.21$ (d, $J=8 \mathrm{~Hz}, 1 \mathrm{H}, \mathrm{CH}), 12.79$ (br, $1 \mathrm{H}$, $\mathrm{NH}, \mathrm{D}_{2} \mathrm{O}$ exchangeable); ${ }^{13} \mathrm{C}$ NMR $\left(100 \mathrm{MHz}, \mathrm{DMSO}-\mathrm{d}_{6}\right): \delta, \mathrm{ppm}=161.27,152.82,148.59$, 132.21, 131.18, 128.99 (2C), 127.59 (2C), 116.69, 104.83, 100.7 4. MS: $\mathrm{m} / \mathrm{z}(\%) 196\left(\mathrm{M}^{+}, 100\right)$, 168 (40), 140 (15), 115 (10), 77 (10).

Synthesis of 2-oxo-6-phenyl-1,2-dihydropyridine-3-carboxamide (6). A mixture of compound 4a $(2.41 \mathrm{~g}, 0.01 \mathrm{~mol})$ in acetic acid $(15 \mathrm{~mL})$ as a solvent was treated with hydrochloric acid $(10 \mathrm{~mL})$, was refluxed for $1 \mathrm{~h}$. The reaction mixture was cooled to room temperature and then poured onto ice-water. The solid, so formed, was collected by filtration and recrystallized from petroleum ether (60-80) to give faint yellow product; yield $80 \%$; mp $270-72{ }^{\circ} \mathrm{C}$. Anal. Calcd for 
$\mathrm{C}_{12} \mathrm{H}_{10} \mathrm{~N}_{2} \mathrm{O}_{2}$ (214.22): C, 67.28; H, 4.71; N, 13.08\% Found: C, 67.19; H, 4.82; N, 13.14\% IR $(\mathrm{KBr}): v_{\max }=3481,3375\left(\mathrm{NH}_{2}\right), 3274(\mathrm{NH}), 1745(\mathrm{CO}), 1658(\mathrm{CO}) ;{ }^{1} \mathrm{H} \mathrm{NMR}(400 \mathrm{MHz}$, DMSO-d 6 ): $\delta, \mathrm{ppm}=6.76\left(\mathrm{br}, 2 \mathrm{H}, \mathrm{NH}_{2}, \mathrm{D}_{2} \mathrm{O}\right.$ exchangeable), $6.80(\mathrm{~d}, J=8 \mathrm{~Hz}, 1 \mathrm{H}, \mathrm{CH}), 7.51$ $7.85(\mathrm{~m}, 5 \mathrm{H}, \mathrm{Ar}-\mathrm{H}), 8.20(\mathrm{~d}, \mathrm{~J}=8 \mathrm{~Hz}, 1 \mathrm{H}, \mathrm{CH}), 12.78\left(\mathrm{br}, 1 \mathrm{H}, \mathrm{NH}, \mathrm{D}_{2} \mathrm{O}\right.$ exchangeable); ${ }^{13} \mathrm{C}$ NMR (100 MHz, DMSO-d $\left.d_{6}\right): \delta, p p m=165.55,163.14,161.27,148.59,145.93,144.20,131.37$, 129.00 (2C), 127.60 (2C), 116.68. MS: m/z (\%) 214 (M+65), 196 (100), 168 (40), 140 (20), 115 (25), 77 (15), 64 (10).

Synthesis of 2-(3-(dimethylamino)-3-phenylallylidene)-malononitrile (7). A mixture of compound 4a $(2.41 \mathrm{~g}, 0.01 \mathrm{~mol})$ and malononitrile $(0.66 \mathrm{~g}, 0.01 \mathrm{~mol})$ in ethanol $(30 \mathrm{~mL})$ as a solvent was treated with piperidine ( 3 drops). The reaction mixture was refluxed for $10 \mathrm{~h}$. The mixture was cooled and then was poured onto ice-water. The solid, so formed, was collected by filtration and recrystallized from benzene to give yellow crystals; yield 95\%; mp 184-86 ${ }^{\circ} \mathrm{C}$. Anal. Calcd for $\mathrm{C}_{14} \mathrm{H}_{13} \mathrm{~N}_{3}$ (223.28): C, 75.31; H, 5.87; N, 18.82\% Found: C, 74.85; H, 5.50; N, $18.99 \%$ IR $(\mathrm{KBr}): v_{\max }=2202(2 \mathrm{CN}) ;{ }^{1} \mathrm{H}$ NMR $\left(400 \mathrm{MHz}, \mathrm{DMSO}-d_{6}\right): \delta, \mathrm{ppm}=2.88(\mathrm{~s}, 3 \mathrm{H}$, $\left.\mathrm{CH}_{3}\right), 3.27$ (s, 3H, $\left.\mathrm{CH}_{3}\right), 5.81(\mathrm{~d}, J=12 \mathrm{~Hz}, 1 \mathrm{H}, \mathrm{CH}), 6.52$ (d, J=12 Hz, 1H, CH), 7.29-7.59 (m, $5 \mathrm{H}, \mathrm{Ar}-\mathrm{H}) ;{ }^{13} \mathrm{C}$ NMR $\left(100 \mathrm{MHz}, \mathrm{DMSO}-d_{6}\right): \delta, \mathrm{ppm}=167.56,157.78,132.64,130.22,129.02$ (2C), 128.50 (2C), 126.88, 118.29, 116.16, 98.63, 56.52, 42.89. MS: m/z (\%) $223\left(\mathrm{M}^{+}, 100\right), 197$ (10), 158 (30), 131 (20), 118 (15), 91 (20), 77 (20).

Synthesis of 5-phenyl-1H-pyrazole (9). A mixture of compound 7 (2.23 g, $0.01 \mathrm{~mol})$ and hydrazine monohydrate $(0.50 \mathrm{~g}, 0.01 \mathrm{~mol})$ in ethanol $(10 \mathrm{~mL})$ as a solvent was refluxed for $5 \mathrm{~h}$ (followed by TIC testing using ethyl acetate: petroleum ether 1:1 until completion). The mixture was cooled and then poured onto ice-water. The solid, so formed, was collected by filtration and recrystallized from petroleum ether (60-80) to give white crystals; yield 90\%; mp 78-80 ${ }^{\circ} \mathrm{C}$. Anal. Calcd for $\mathrm{C}_{9} \mathrm{H}_{8} \mathrm{~N}_{2}$ (144.128): C, 74.98; H, 5.59; N, 19.43\% Found: C, 74.63; H, 5.19; N, $19.25 \% \operatorname{IR}(\mathrm{KBr}): v_{\max }=3165(\mathrm{NH}) ;{ }^{1} \mathrm{H} \mathrm{NMR}\left(400 \mathrm{MHz}, \mathrm{CDCl}_{3}\right): \delta, \mathrm{ppm}=6.64(\mathrm{~d}, J=4 \mathrm{~Hz}$, 1H, CH), 7.33-7.45 (m, 3H, Ar-H), 7.64 (d, J=4 Hz, 1H, CH), 7.77-7.80 (m, 2H, Ar-H), 8.53 (br, $1 \mathrm{H}, \mathrm{NH}, \mathrm{D}_{2} \mathrm{O}$ exchangeable); ${ }^{13} \mathrm{C} \mathrm{NMR}\left(100 \mathrm{MHz}, \mathrm{CDCl}_{3}\right): \delta, \mathrm{ppm}=149.36,133.44$, 132.32, 129.00 (2C), 128.27, 126.03 (2C), 102.88. MS: m/z (\%) $144\left(\mathrm{M}^{+}, 100\right), 115$ (25), 89 (15), 77 (20), 63 (15).

Synthesis of 2-oxo-6-phenyl-2H-pyran-3-carbonitrile (10). A mixture of compound 1a $(1.75 \mathrm{~g}, 0.01 \mathrm{~mol})$ and cyanoacetic acid $(0.85 \mathrm{~g}, 0.01 \mathrm{~mol})$ in acetic anhydride $(15 \mathrm{~mL})$ as a solvent, was refluxed for $3 \mathrm{~h}$ (followed by TIC testing using ethyl acetate: petroleum ether 1:1 until completion). The mixture was cooled and then poured onto ice-water. The solid, so formed, was collected by filtration and recrystallized from ethanol to give dark yellow product; yield 80\%; mp 178-80 ${ }^{\circ} \mathrm{C}$. Anal. Calcd for $\mathrm{C}_{12} \mathrm{H}_{7} \mathrm{NO}_{2}$ (197.19): C, 73.09; H, 3.58; N, 7.10\% Found: C, 72.64; H, 3.15; N, 7.44\% IR (KBr): $\mathrm{v}_{\max }=2225(\mathrm{CN}), 1727$ (CO); ${ }^{1} \mathrm{H}$ NMR (400 MHz, DMSO$\left.d_{6}\right): \delta, p p m=7.35(\mathrm{~d}, J=8 \mathrm{~Hz}, 1 \mathrm{H}, \mathrm{CH}), 7.56-7.99(\mathrm{~m}, 5 \mathrm{H}, \mathrm{Ar}-\mathrm{H}), 8.50(\mathrm{~d}, J=8 \mathrm{~Hz}, 1 \mathrm{H}, \mathrm{CH})$; ${ }^{13} \mathrm{C}$ NMR $\left(100 \mathrm{MHz}, \mathrm{DMSO}-d_{6}\right): \delta, \mathrm{ppm}=164.31,158.18,154.08,132.64,129.86,129.43(2 \mathrm{C})$, 126.51 (2C), 115.03, 102.24, 98.49. MS: m/z (\%) $196\left(\mathrm{M}^{+}, 90\right), 168$ (100), 139 (20), 104 (50), 76 (50). 
Synthesis of 3-(dimethylamino)-6-phenyl-2H-pyran-2-one (12). A mixture of compound 1a $(1.75 \mathrm{~g}, 0.01 \mathrm{~mol})$ and ethylcyanoacetate $(1.13 \mathrm{~g}, 0.01 \mathrm{~mol})$ in ethanol $(20 \mathrm{~mL})$ as a solvent, was refluxed for $4 \mathrm{~h}$ (followed by TIC testing using ethyl acetate : petroleum ether $1: 1$ until completion). The mixture was cooled to room temperature and then poured onto ice-water. The solid, so formed, was collected by filtration and recrystallized from ethanol to give faint yellow product; yield 85\%; mp 93-95 ${ }^{\circ} \mathrm{C}$. Anal. Calcd for $\mathrm{C}_{13} \mathrm{H}_{13} \mathrm{NO}_{2}$ (215.25): C, 72.54; H, 6.09; N, 7.51\% Found: C, 72.48; H, 6.15; N, 7.59\% IR (KBr): $\mathrm{v}_{\max }=1657$ (CO); ${ }^{1} \mathrm{H}$ NMR (400 MHz, DMSO-d $)_{6}: \delta, p p m=2.91\left(\mathrm{~s}, 3 \mathrm{H}, \mathrm{CH}_{3}\right), 3.14\left(\mathrm{~s}, 3 \mathrm{H}, \mathrm{CH}_{3}\right), 5.81(\mathrm{~d}, J=12 \mathrm{~Hz}, 1 \mathrm{H}, \mathrm{CH}), 7.43-$ $7.48(\mathrm{~m}, 3 \mathrm{H}, \mathrm{Ar}-\mathrm{H}), 7.71(\mathrm{~d}, J=12 \mathrm{~Hz}, 1 \mathrm{H}, \mathrm{CH}), 7.87-7.89$ (m, 2H, Ar-H); ${ }^{13} \mathrm{C} \mathrm{NMR}(100 \mathrm{MHz}$, DMSO-d $\left.d_{6}\right): \delta, p p m=185.70,154.19,152.83,140.23,130.78,128.15$ (2C), 127.17 (2C), 90.89, 83.21, 44.51, 37.12. MS: m/z (\%) $214\left(\mathrm{M}^{-1}, 80\right), 185$ (50), 157 (25), 134 (15), 108 (100), 93 (15), $80(80), 67(10), 53(20)$.

Synthesis of 3-oxo-3-phenyl-2-(2-phenylhydrazono)-propanal (17). A solution of compound $12(2.15 \mathrm{~g}, 0.01 \mathrm{~mol})$ in ethanol $(50 \mathrm{~mL})$ was treated with sodium acetate $(5 \mathrm{~g})$. Aniline diazonium chloride (prepared according to the standard literature procedures from aniline $(0.93 \mathrm{~g}, 0.01 \mathrm{~mol})$, which was added gradually with stirring to a cooled mixture of concentrated hydrochloric acid, and sodium nitrite). After complete addition of the diazonium salt, the reaction mixture was kept at room temperature for one hour. The solid, so formed, was collected by filtration and crystallized from benzene to give orange dark crystals; yield $90 \%$; mp 96-97 ${ }^{\circ} \mathrm{C}$ (literature mp $96{ }^{\circ} \mathrm{C}$ ). Anal. Calcd for $\mathrm{C}_{15} \mathrm{H}_{12} \mathrm{~N}_{2} \mathrm{O}_{2}$ (252.27): C, 71.42; H, 4.79; N, 11.10\% Found: C, 71.63; H, 4.47; N, 11.37\% IR (KBr): $v_{\max }=3431(\mathrm{NH}), 1645(\mathrm{CO}), 1631(\mathrm{CO}) ;{ }^{1} \mathrm{H}$ NMR (400 MHz,CDCL 3 ): $\delta, \mathrm{ppm}=7.21-7.99(\mathrm{~m}, 10 \mathrm{H}, \mathrm{Ar}-\mathrm{H}), 10.20$ (s, 1H, CHO), 10.76 (br, $1 \mathrm{H}, \mathrm{NH}, \mathrm{D}_{2} \mathrm{O}$ exchangeable); ${ }^{13} \mathrm{C} \mathrm{NMR}\left(100 \mathrm{MHz}, \mathrm{CDCL}_{3}\right): \delta, \mathrm{ppm}=191.98,189.75,141.31$, 137.23, 132.50, 132.39, 130.58 (2C), 129.97 (2C), 127.97 (2C), 126.68, 116.77 (2C); MS: m/z (\%) $252\left(\mathrm{M}^{+}, 60\right), 223(20), 132$ (70), 105 (90), 93(60), 77 (100), 65 (30).

\section{Acknowledgements}

The authors are grateful to Kuwait University Research Administration for the financial support of project SC10/06 and for SAF facilities project GS01/02 and GS03/01.

\section{References}

1. Riyadh, S. M.; Abdelhamid, I. A.; Al-Matar, H. M.; Hilmy N. M.; Elnagdi, M. H. Heterocycles 2008, 75, 1849.

2. Al-Mousawi, S. M.; Moustafa, M. S.; Elnagdi, M. H. Arkivoc 2008, (x), 17.

3. Abdelkhalik, M. M.; Elnagdi, M. H. Synth. Commun. 2002, 32, 159.

4. El-Taweel, F. M. A.; Elnagdi, M. H. J. Heterocycl. Chem. 2001, 38, 981. 
5. Al-Saleh, B.; Al-Awadi, N.; Al-kandari, H.; Abdel-Khalik, M. M.; Elnagdi, M. H. J. Chem. Res. (S), 16; (M), 2000, 201.

6. Agamy, S. M.; Abdel-Khalik, M. M.; Mohamed, M. H.; Elnagdi, M. H. Z. Naturforschung 2001, 56b, 1074.

7. El-Enezi, A.; Al-Saleh, B.; Elnagdi, M. H. J. Chem. Res. (S), 4; (M). 1997, 110.

8. Almazroa, S.; Elnagdi, M. H.; Salah El-Din, A. M. J. Heterocycl. Chem. 2004, 41, 267.

9. Al-Saleh, B.; Makhseed, S.; Hassaneen, H. M. E.; Elnagdi, M. H. Synthesis 2006, 59.

10. Al-Mousawi, S. M.; Abdel-Khalik, M. M.; El-Sherbiny, S.; John, E.; Elnagdi, M. H. J. Heterocycl. Chem. 2002, 38, 949.

11. Cimarelli, C.; Palmieri, G. Rec. Res. Dev. Org. Chem. 1997, 1, 179.

12. Kralj, D.; Novak, A.; Dahmann, G.; Groselj, U.; Meden, A.; Svete, J. J. Comb. Chem. 2008, $10,664$.

13. Svete, J. J. Heterocycl. Chem. 2005, 42, 361.

14. Wagger, J.; Groselj, U.; Meden, A.; Svete, J.; Stanovnik, B. Tetrahedron 2008, 64, 2801.

15. Yavari, I.; Hossaini, Z.; Sabbaghan, M. Tetrahedron Lett. 2008, 49, 844.

16. Al-Omran, F.; Al-Awadi, N.; ElKhair, A. A.; Elnagdi, M. H. OPPI 1997, 29, 285.

17. Al-Omran, F.; Al-Awadi, N.; Abdel Khalik, M. M.; Kaul, K.; ElKhair, A. A.; Elnagdi, M. H. J. Chem. Res. (S) 84; (M) 1997, 601.

18. Gorobets, N. Y.; Yousefi, B. H.; Belaj, F.; Kappe, C. O. Tetrahedron 2004, 60, 8633.

19. Alnajjar, A. A.; Abdelkhalik, M. M.; Al-Enezi, A.; Elnagdi, M. H. Molecules 2009, 14, 68.

20. CCDC 720655 contains the supplementary crystallographic data for compound $\mathbf{4 a}$ in this paper. These data can be obtained free of charge from the Cambridge Crystallographic Data Centre via www.ccdc.cam.ac.uk

21. CCDC 721579 contains the supplementary crystallographic data for compound 7 in this paper. These data can be obtained free of charge from the Cambridge Crystallographic Data Centre via www.ccdc.cam.ac.uk

22. Gérard, A. L.; Bouillon, A.; Mahatsekake, C.; Collo, V.; Raul, V. Tetrahedron Lett. 2006, 47, 4665. 